\title{
Novel Multi-Functional Dental Cement for Enamel Remineralization and Anti-Cariogenic Bacteria Activity
}

\author{
Yukari Shinonaga ${ }^{1 *}$, Kenji Arita $^{1}$, Rie Imataki $^{2}$, Michiko Takemura $^{2}$, Chikoto Nagaishi ${ }^{2}$, Keiichi $^{2}$ \\ Kagami $^{2}$, Takako Nishimura ${ }^{1}$, Yoko Abe ${ }^{1}$, Sho Aoki ${ }^{2}$, Masae Okuno ${ }^{2}$ and Kyoko Harada ${ }^{1}$
}

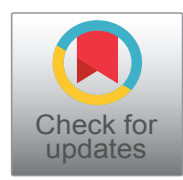

${ }^{1}$ Department of Pediatric Dentistry, School of Dentistry, Osaka Dental University, Japan

${ }^{2}$ Graduate School of Dentistry (Department of Pediatric Dentistry), Osaka Dental University, Japan

*Corresponding author: Yukari Shinonaga, Department of Pediatric Dentistry, School of Dentistry, Osaka Dental University, 1-5-17, Otemae, Chuo-ku, Osaka 540-0008, Japan, Tel: +81-6-6910-1515, Fax: +81-6-6910-1038

\begin{abstract}
Objective: This experimental study evaluated the release of fluoride and several minerals related to remineralization from a novel functional cement, apatite ionomer cement (AIC), and its anti-bacterial properties compared with glass ionomer cement (GIC) and surface pre-reacted glass-ionomer filler containing composite resin (giomer).

Materials and methods: Conventional GIC (Fuji III, GC Co., Tokyo, Japan) was used as the control and fundamental materials. In the AIC powder, $28 \%$ wt of GIC powder was replaced with spherical-shaped hydroxyapatite powder. The giomer, BeautiSealant (Shofu Co., Kyoto, Japan), was used as a positive control. Each specimen was immersed in deionized water and analyzed by inductively coupled plasma atomic emission spectrometry (for $\mathrm{Al}, \mathrm{Si}, \mathrm{P}, \mathrm{Ca}$ and $\mathrm{Sr}$ ) and a fluoride-selective electrode. Antibacterial activity against Streptococcus mutans was evaluated using the adenosine-5'- triphosphate luminescence method.

Results: Concentrations of released ions from AIC specimens were significantly higher than those from GIC and giomer specimens, except for Sr. Regarding antibacterial activity, luminescence intensity of the AIC group (27.2 \pm 12.6 $\mathrm{RLU})$ was significantly lower than that of the giomer group (787.4 \pm 176.1 RLU).

Conclusion: It was concluded that AIC could be a most suitable material for pit and fissure sealant for enamel remineralization and anti-cariogenic and -bacterial activity.
\end{abstract}

\section{Keywords}

Antibacterial property, Fluoride ion release, Glass ionomer cement, Hydroxyapatite, S-PRG

\section{Introduction}

The pathophysiological processes of dental caries lead to mineral loss, which occurs as a result of an imbalance between enamel or dentin demineralization and remineralization. Recently, caries management has increasingly adopted more conservative approaches against conventional surgical treatment, depending on the early detection of white-spot lesions and the use of remineralizing agents to reverse or arrest caries lesion progression. Fluoride promotes remineralization and inhibits demineralization of dental hard tissue [1].

Fissure sealants are materials placed in the pits and fissures of teeth in order to prevent or arrest the development of dental caries. Their effectiveness has been demonstrated through numerous studies and summarized in a systematic review [2]. However, several factors, such as operator skill, sealant material, fissure type, enamel quality, and teeth eruption phase influence the optimal placement of fissure sealants [3]. Moreover, the removal of debris and biofilm, and the setting of sealant materials at the bottom of the fissure are complicated in the case of a deep and narrow fissure [4]. Furthermore, it is more desirable for the sealant material to have good antimicrobial and remineralization properties than good sealing properties.

Fissure sealants are normally composite resin or glass ionomer cement (GIC) materials. In a systematic review [2] comparing the results of eight studies of composite resin-based and GIC-based sealants, three stud- 
ies reported an improved caries-preventive effect for composite resin-based sealants, two studies reported a better caries-preventive effect for GIC-based sealants, while three studies found no significant difference between composite resin-based and GIC-based sealants. Another review concluded that there was no consistent difference in caries-preventive effect between composite resin- and GIC-based sealants [5].

GIC, introduced in the 1970s by Wilson and Kent [6], has been widely used as a fluoride-releasing material in dental clinics [7-9]. GIC materials are mineral-based, adhere to the tooth surface, and facilitate post-eruptive mineral uptake in the enamel. Owing to these desirable properties, GIC products have been used as sealing materials in the pits and fissures of teeth in order to prevent or arrest the development of dental caries. GIC materials have been found to exert a cariostatic effect even after they had disappeared macroscopically, and this effect might be based on remnants of the cement in the fissure as well as increased levels of fluoride ions on the enamel surface [10]. Researchers recently indicated that GIC could be effectively used, not only for occlusal initial caries, but also for proximal caries and demonstrated that fluoride released from GIC restorations inhibited the progression of proximal caries in adjacent teeth [11]. Proximal sealing with GIC directly could also manage initial proximal caries [12].

However, the use of GIC has been limited due to its inferior mechanical strength, wear resistance, and sensitivity to initial moisture $[13,14]$. Therefore, several researchers have attempted to strengthen the mechanical properties of GICs [15-17]. Hybrid materials that combine GIC and a composite resin, such as resin-modified GICs, polyacid-modified resin composites (compomers), and giomers have been developed. In particular, giomers, a type of composite resin, have been introduced in the latest development of fluoride-releasing materials. They contain a surface pre-reacted glass-ionomer (S-PRG) filler in the resin matrix and possess anti-bacterial activity due to their ion release properties [1821]. However, some researchers have suggested that the properties of giomer-products are less conspicuous than those of S-PRG fillers [22,23].

In contrast, we previously reported that a novel material, apatite ionomer cement (AIC), with a GIC matrix modified by hydroxyapatite particles, not only improves flexural and compressive strength, but also increases the quantity of fluoride ions released [24,25]. We also demonstrated that it possesses antimicrobial activity against cariogenic bacteria [26].

The purpose of the present study was to evaluate and compare the ion release and antibacterial properties of a novel material (AIC) with GIC and giomer.

\section{Materials and Methods}

\section{Materials}

A conventional GIC pit and fissure sealant, Fuji III (GC
Co., Tokyo, Japan), was used for the control GIC (GIC group) and as the base material for the AIC group. Based on preliminary experiments, AIC powder contained $28 \%$ wt spherical-shaped hydroxyapatite particles (HApS) comprising Fuji III glass powder and HApS. GIC and AIC powders were mixed with Fuji III liquid at a powder/liquid ratio of 1.2, according to the manufacturer's recommendation. All the cements were mixed in 60 seconds. In addition, BeautiSealant (Shofu Co., Kyoto, Japan), a giomer, was used for a positive control (giomer group). The specimens of giomer group light-cured in $20 \mathrm{sec}-$ onds by Highly concentrated light in a cordless handpiece, Pencure (J. Morita Co., Osaka Japan).

\section{Fluoride ion release test}

Five cylindrical specimens $(10 \mathrm{~mm}$ in diameter $\times 2$ $\mathrm{mm}$ in thickness) were prepared for each of the GIC, AIC, and giomer groups using plastic split mold. The samples were individually suspended by a cotton thread in $18 \mathrm{ml}$ of distilled water in sealed containers and were stored at $37^{\circ} \mathrm{C}$. For the measurements, each disk was removed from the water, washed by immersion in $2 \mathrm{ml}$ of water, dried on filter paper, and immediately immersed in 18 $\mathrm{ml}$ of fresh distilled water for further equilibration. A volume of $2 \mathrm{ml}$ of total ionic strength adjustment buffer solution (TISAB III, Thermo Fisher Scientific, Beverly, MA, USA) was added to the water sample. The fluoride ion concentration was measured daily for 7 days using a fluoride-selective electrode (6561-10 C, Horiba Ltd., Kyoto, Japan) connected to an ion analyzer D-53 (Horiba Ltd.).

\section{Elemental analysis by inductively coupled plas- ma-atomic emission spectroscopy}

Four cylindrical specimens $(10 \mathrm{~mm}$ in diameter $\times 2$ $\mathrm{mm}$ in thickness) for each of the three groups were prepared in the same manner as in the fluoride ion release test. The samples were individually suspended by a cotton thread in $20 \mathrm{ml}$ of distilled water in sealed containers and were stored at $37^{\circ} \mathrm{C}$ for 7 days. The amounts of $\mathrm{Al}, \mathrm{Si}, \mathrm{P}, \mathrm{Ca}$ and $\mathrm{Sr}$ released from the specimens were subsequently measured by inductively coupled plasma-atomic emission spectroscopy (ICPS-8100, Shimadzu Co., Kyoto, Japan).

\section{Anti-bacterial activity test}

For the antibacterial activity test, six samples measuring $10 \mathrm{~mm}$ in diameter $\times 2 \mathrm{~mm}$ in thickness per group were prepared in a plastic split mold. Antibacterial tests were performed using a bioluminescence method; The adenosine-5'-triphosphate (ATP) luminescence method according to a preliminary study [26]. This method utilizes the luminescent mechanism of firefly luciferase; Fireflies generate light by creating a reaction inside their bodies between their own ATP and the enzyme luciferase. The presence of ATP can be considered proof of the presence of a living organism [27]. The amount of bacterial contamination around the specimens was evaluat- 


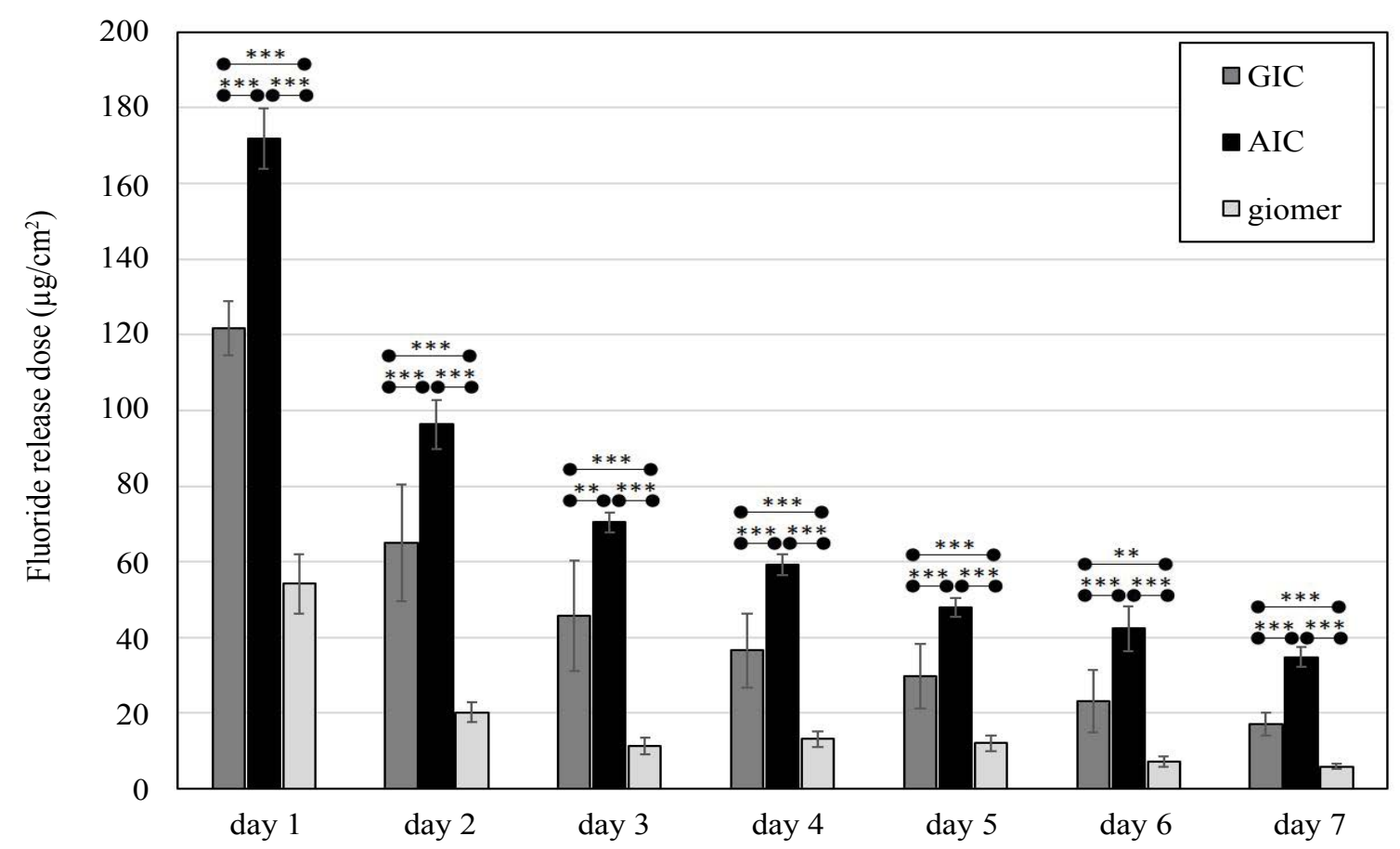

Figure 1: Fluoride ion release from GIC, AIC, and giomer into deionized water.

GIC: Glass ionomer cement; AIC: Apatite ionomer cement; Analysis of variance/Tukey: ${ }^{* *} p<0.01,{ }^{* * *} p<0.001$.

ed by measuring the luminescence level of the ATP-luciferase reaction. Streptococcus mutans ATCC25175 was used as the test bacteria. The $S$. mutans suspension was spectrophotometrically $(660 \mathrm{~nm})$ standardized to a concentration of $8 \times 10^{6} \mathrm{CFU} / \mathrm{ml}$. Samples were immersed in a $S$. mutans suspension and were then incubated at $37^{\circ} \mathrm{C}$ for 4 hours. Subsequently, samples were removed from the $S$. mutans suspensions and diluted with physiologic saline, and the ATP luminescence intensity of the suspension was evaluated using a luciferin-luciferase ATP assay reagent kit (Lucifer HS Set, product code: 60315, Kikkoman Biochemifa Co., Chiba, Japan) and a luminometer (Lumitester C-110, Kikkoman Co.), according to the manufacturer's instructions. The luminescence intensity was expressed in relative luminescence units (RLU). In addition, the luminescence intensity of a 'no-sample group' comprising an $S$. mutans suspension without the immersion of any sample, was measured in order to observe the increase of $S$. mutans before incubation (original $S$. mutans suspension) and after 4 hours of incubation.

\section{Statistical analysis}

Data are presented as mean \pm standard deviation. Firstly, homoscedasticity was confirmed by the Bartlett's test. Then data were analyzed by one-way analysis of variance and Tukey's test (Kaleida Graph 4.00, Synergy Software, Reading, PA, USA), with $p$ values less than 0.05 being considered statistically significant. The confidence interval was set at $95 \%$.

\section{Results}

\section{Fluoride release properties}

GIC, AIC and giomer daily fluoride ion release amounts are shown in Figure 1. Release amounts were significantly larger in the AIC group than in the GIC group on all experimental days $(p<0.01)$. Moreover, fluoride release amounts from giomer specimens were significantly lower than those from AIC and GIC specimens on all experimental days $(p<0.01)$.

\section{Multi-mineral release properties}

Figure 2 shows the results of inductively coupled plasma-atomic emission spectroscopy measurements in the GIC, AIC and giomer groups. Al and Si release from AIC was significantly greater than that from GIC and giomer. The release of $P$ from AIC was significantly greater than that from GIC. Furthermore, $\mathrm{P}$ was not detected in the water-immersed giomer group. Moreover, Ca was detected in water-immersed AIC group, but not in GIC or giomer groups. The release of $\mathrm{Sr}$ from giomer was significantly greater than that from GIC. There was no significant difference in $\mathrm{Sr}$ release between the AIC and giomer groups.

\section{Anti-bacterial properties}

The luminescence intensities of the GIC, AIC, giomer and no-sample groups before incubation and after 4 hours incubation were compared. The luminescence intensity of the $S$. mutans suspension without specimens increased significantly after 4 hours of incubation, and the luminescence intensities of the AIC and GIC groups decreased significantly after 4 hours of incubation. Moreover, the intensities of the AIC and GIC groups decreased significantly compared with the giomer and no-sample groups. The intensity of the giomer group was approximately the same as that of the no-sample 

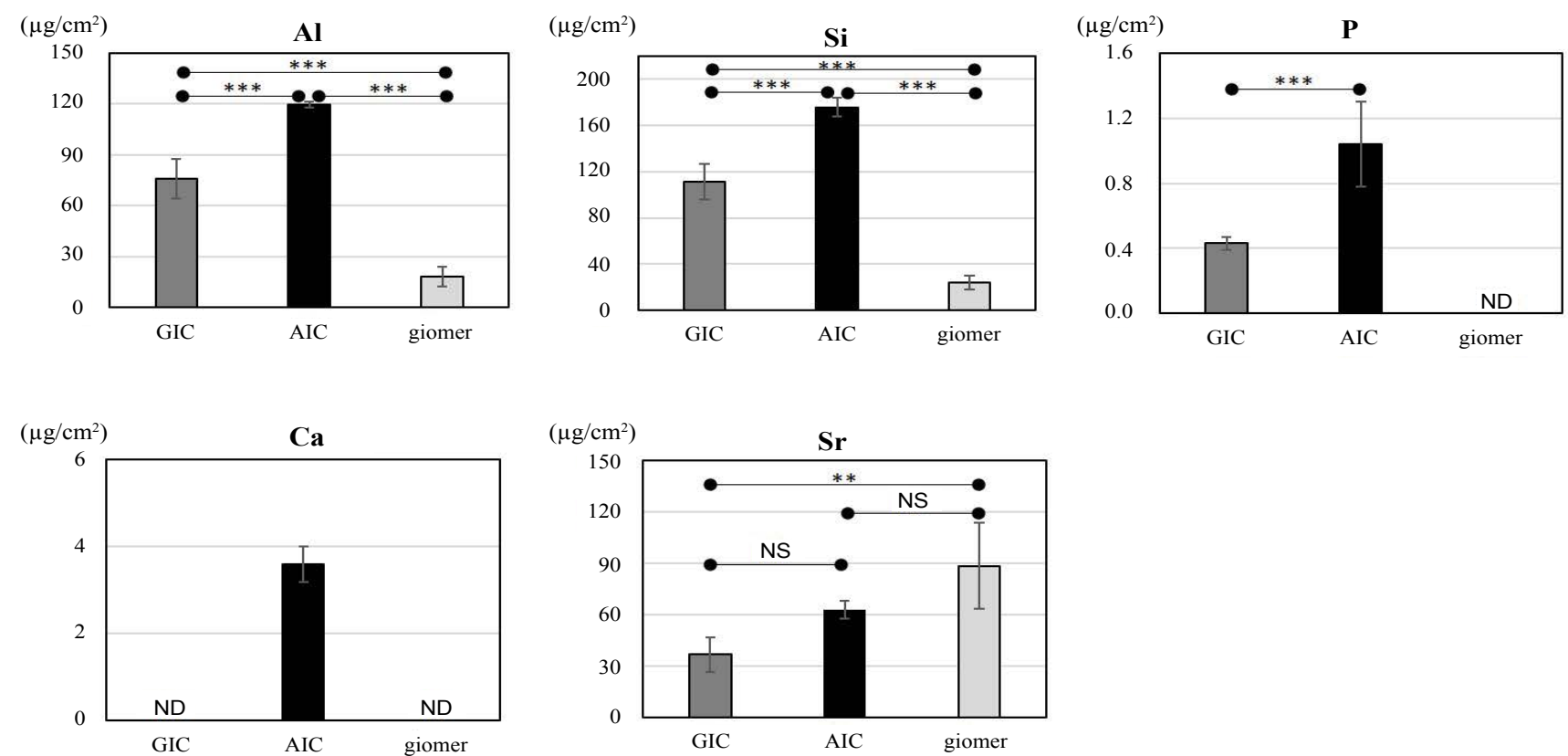

Figure 2: Ion (Al, Si, P, Ca, and Sr) release from GIC, AIC, and giomer into deionized water.

GIC: Glass ionomer cement; AIC: Apatite ionomer cement; ND: Not detected; Analysis of variance/Tukey: ${ }^{* *} p<0.01,{ }^{* * *} p<$ 0.001 ; NS: Not significant difference.

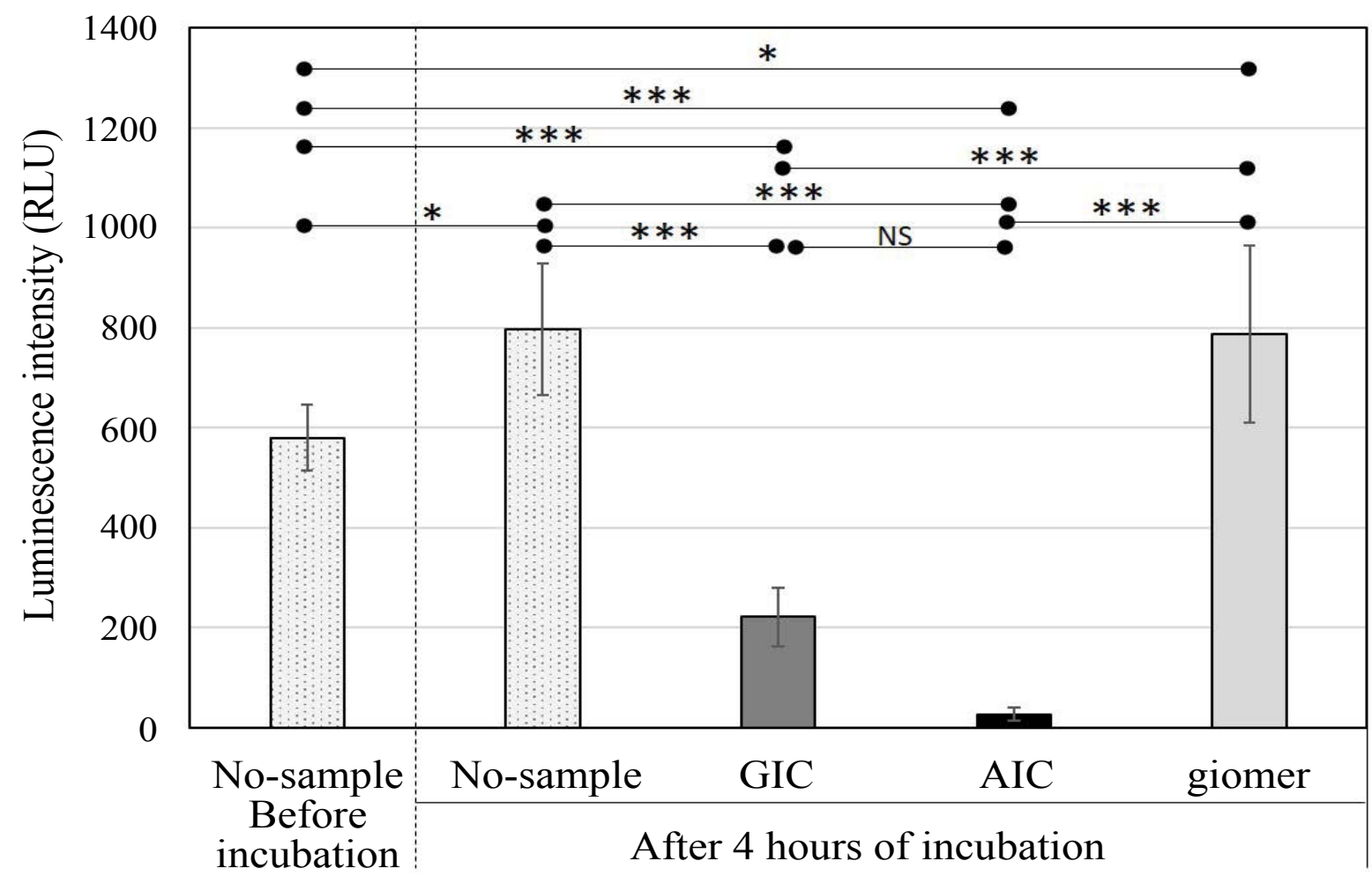

Figure 3: Luminescence intensity of $S$. mutans suspensions with GIC, AIC, and giomer specimens and without specimens before and after incubation.

GIC: Glass ionomer cement; AIC: Apatite ionomer cement; Analysis of variance/Tukey: ${ }^{*} p<0.05,{ }^{* * *} p<0.001$; NS: Not significant difference.

group, which is considered to not exhibit effective antibacterial activity (Figure 3 ).

\section{Discussion}

This experimental study evaluated the release of several remineralization-related ions from a novel functional cement, AIC, and its anti-bacterial properties compared with GIC and giomer. We found that AIC had a significantly greater fluoride ion release property compared with GIC and giomer. Although the mechanism of fluoride release from GIC is not yet fully understood, Hatibovic-Kofman, et al. [28] demonstrated that 
the quantity of released fluoride is positively correlated with the quantity of fluoride originally incorporated in the GIC product. Fluoride in GIC originates from fluoro-alminosilicate glass. In the present study, AIC specimens contained less fluoro-alminosilicate glass powder than the GIC specimens because $28 \%$ wt of glass powder was replaced with HAp powder. Lewis, et al. [29] demonstrated that HAp reacts with acidic storage media from glass-ionomer cements to take up fluoride, regardless of whether or not the fluoride forms a complex with any other chemical species. In previous studies $[27,30]$, we showed that innumerable nano-HApS from porous spherical-shaped HAp were dispersed in the AIC matrix. It is inferred from these findings that the mechanism of increased fluoride ion release from AIC was due to nano-HApS dispersal in the matrix layer acting as a reservoir of fluoride ions and the release of stored fluoride ions from the cement bulk. Moreover, it was demonstrated that the release of fluoride ions from GIC containing cellulose, which is incapable of reacting with GIC, was also higher compared with that from GIC [31]. A second potential reason for the increase in the amount of fluoride ions released compared with GIC may be due to greater amount of polyacrylic acid liquid in AIC, which can react with fluoro-alminosilicate glass. If the latter reason is valid, it will also explain the increase in releases of $\mathrm{Al}, \mathrm{Sr}$, and $\mathrm{Si}$ in the present study. However, further research is required to ascertain these reasons.

In the present study, $\mathrm{Al}$ and $\mathrm{Si}$ releases from $\mathrm{AIC}$ were also superior to those from GIC and giomer. McCann [32] investigated the effects of various metal ions, especially $\mathrm{Al}$, on the uptake and retention of fluoride by human enamel. Moreover, some enamel dissolution experiments demonstrated that Al reacts with, and is incorporated into, enamel, and also possesses cariostatic properties [33-35]. While Al release is required to inhibit enamel demineralization, it has several well-known toxic effects in humans, including adverse effects on the central nervous system, skeleton, and hematopoietic system [36]. However, Nicholson, et al. [37] calculated that if a GIC filling dissolved completely over 5 years, an adult patient would only consume an extra $0.5 \%$ of the recommended maximum $\mathrm{Al}$ intake. This led to the conclusion that the release of Al from any type of GIC placed in the mouth is unlikely to pose a health hazard. In the present study, the release of Al from AIC was significantly higher than that from GIC. However, this increase was not considered to be directly detrimental to patient health.

Besinis, et al. suggested that Si has the ability to penetrate dentin, remain embedded within the collagen matrix, and provide a suitable scaffold for the remineralization of dentin [38]. The present findings indicate that the higher Si release from AIC leads to the promotion of tooth structure remineralization. In addition, the $\mathrm{Sr}$ release from giomer was superior to that from GIC, but there was no significant difference between AIC and giomer groups. Commercial GICs, including the one used in the present study, contain glass with complete substitution of Ca by $\mathrm{Sr}$; This is advantageous because $\mathrm{Sr}$ has known anti-cariogenic properties [39]. In addition, Featherstone, et al. [40] reported that when $\mathrm{Sr}$ and $\mathrm{F}$ were incorporated together they improved apatite crystallinity and were associated with marked reductions in the acid reactivity of synthetic carbonated apatite, which is observed in dental enamel during the early stages of dental decay. The release of fluoride and $\mathrm{Sr}$ from AIC could therefore promote the remineralization of early caries lesion. On the other hand, $\mathrm{Ca}$, which was dislodged from the main element of GIC glass, has been demonstrated to enhance the formation of the GIC matrix, increasing surface hardness [41]. Therefore, $\mathrm{Ca}$ is necessary to improve both the mechanical strength and remineralization property of GIC. The addition of HAp to AIC could potentially represent a source of $\mathrm{Ca}$ that improves the mechanical strength of GIC because we have previously shown that AIC has significant high mechanical strength compared with GIC [24-26].

The anti-bacterial properties of AIC and GIC was demonstrated in the present study. Moreover, the luminescence intensities of $S$. mutans suspensions containing immersed GIC and AIC were compared through both multiple comparisons and $t$-test. Results revealed that the luminescence intensity of AIC was decreased significantly compared with that of GIC after incubation for 4 hours. The present study was designed to compare the bacterial increase in $S$. mutans suspensions with and without specimens (no-sample group). Comparison of the luminescence intensity before and after incubation revealed no significant difference between the $S$. mutans suspensions containing giomer specimens and those without specimens. This suggests that giomer sealant is unable to inhibit $S$. mutans growth.

In the present study, we compared anti-bacterial properties between AIC and giomer (S-PRG filler containing composite resin). S-PRG filler particles are formed by an acid-based reaction of fluoroalumino-silicate glass with polyacrylic acid, and six types of ions $\left(\mathrm{Na}^{+}, \mathrm{Sr}^{2+}, \mathrm{SiO}_{3}{ }^{2-}, \mathrm{Al}^{3+}, \mathrm{BO}_{3}{ }^{3-}\right.$ and $\left.\mathrm{F}^{-}\right)$, have been reported to be released from S-PRG filler [22,42]. The mechanism of ion release from S-PRG filler is believed to relate to the presence of a glass ionomer layer around the glass core filler. Several researchers have demonstrated that various ions released from S-PRG filler inhibit dentin demineralization $[43,44]$, reduce plaque formation [45], and inhibit oral bacteria growth [18-21]. However, in the present study, S-PRG filler containing pit and fissure sealant material was found to have a less potent anti-bacterial property compared with that of GIC and AIC. An, et al. also reported similar results in a comparison with other composite resin sealants [23].

Most studies on the antibacterial properties of 
S-PRG filler have used elute from the S-PRG filler particle itself or materials containing a number of S-PRG filler particles as experimental samples and have reported higher S-PRG filler content leading to stronger antibacterial properties [18-21]. An, et al. suggested that BeautiSealant, which formed the giomer group in the present study, did not contain sufficient amounts of S-PRG filler to have an antibacterial effect [23]. Fujimoto, et al. also concluded that ion release from S-PRG filler particles was influenced by the mixing ratio of the solution [22]. Moreover, Saku, et al. used blocks of Beautifil II (Shofu Inc.), S-PRG filler containing composite resin for restoration, and evaluated its antibacterial activity in comparison with other commercial composite resins lacking S-PRG filler [45]. They reported no significant difference in antibacterial effect among the three composite resins. Another study demonstrated that the concentration of ions released by S-PRG-filled resin-based composite appeared to be insufficient for inhibiting bacterial growth [46]. Based on these considerations, the amounts of various effective ions eluted from composite resin containing S-PRG filler might be less than that from the filler itself, although S-PRG filler itself has a superior ion releasing property.

The present results suggest that the anti-bacterial effect of AIC caused superior fluoride and other mineral release properties. However, there is still insufficient evidence to accurately determine the antibacterial property. Further microbiological assessments are required to understand if the antibacterial effect is due to a change in $\mathrm{pH}$ of media, lack of adhesion, metabolism inhibition, or mortality effect and to confirm the present results.

In this study, we selected a commercial GIC and giomer for pit and fissure sealant in order to evaluate the effectiveness of AIC application in non-surgical and conservative treatment for initial caries lesion, such as pit and fissure sealant and proximal sealant. AIC proved to be superior to GIC in mechanical strength; The flexural strength of GIC and AIC has been reported to be $5.84 \pm$ $1.31 \mathrm{MPa}$ and $13.78 \pm 1.58 \mathrm{MPa}$, respectively [26]. In the preliminary study, the flexural strength of the giomer used was $52.80 \pm 6.71 \mathrm{MPa}$. It was shown to still be inferior to Giomer which is a type of composite resin. However, AIC was observed to release a sufficient quantity of ions to produce an antibacterial effect equivalent to that of giomer, and it could be concluded that AIC is the most suitable material for conservative treatment of initial caries lesions compared with surgical approaches.

\section{Conclusions}

The present study was conducted in order to determine the amounts of ions (fluoride, $\mathrm{Al}, \mathrm{Si}, \mathrm{Ca}, \mathrm{P}$ and $\mathrm{Sr}$ ) released from GIC, AIC and giomer. The results revealed that AIC released significantly greater amounts of fluoride, $\mathrm{Al}, \mathrm{Si}$ and $\mathrm{Ca}$ than $\mathrm{GIC}$ and giomer, and had the greatest antibacterial effect. It was concluded that AIC can release sufficient amounts of several types of ions to induce an antibacterial effect compared with giomer. It was concluded that AIC could be a most suitable material for pit and fissure sealant for enamel remineralization and anti-cariogenic and -bacterial activity. We will further focus on not only remineralization effect but also demineralization suppressing effect for teeth in the future research.

\section{Acknowledgments}

The present study was supported by Grants-in-Aid for Scientific Research 'KAKENHI' from the Japan Society for the Promotion of Science [JSPS Grant Numbers 16K11820, 17K18247, and 17K17200]. The authors would like to thank Dr. Szu-Yu Chiu, D.D.S., PhD. at the Department of Oral Hygiene, Shu-Zen Junior College of Medicine and Management, Kaohsiung, Taiwan (R.O.C.), for her helpful examination and discussions. We would like to thank Editage (www.editage.jp) for English language editing.

\section{Conflicts of Interest}

There are no potential conflicts of interest to be disclosed.

\section{References}

1. Featherstone JD, Glena R, Shariati M, Shields CP (1990) Dependence of in vitro demineralization of apatite and remineralization of dental enamel on fluoride concentration. $J$ Dent Res 69: 620-625.

2. Ahovuo-Saloranta A, Hiiri A, Nordblad A, Mäkelä M, Worthington HV (2008) Pit and fissure sealants for preventing dental decay in the permanent teeth of children and adolescents. Cochrane Database Syst Rev.

3. Simonsen RJ (2002) Pit and fissure sealant: Review of the literature. Pediatr Dent 24: 393-414.

4. Weerheijm KL, de Soet JJ, van Amerongen WE, de Graaff J (1992) Sealing of occlusal hidden caries lesions: An alternative for curative treatment? ASDC J Dent Child 59: 263-268.

5. Beiruti N, Frencken JE, van't Hof MA, van Palenstein Helderman WH (2006) Caries-preventive effect of resin-based and glass ionomer sealants over time: A systematic review. Community Dent Oral Epidemiol 34: 403-409.

6. Wilson AD, Kent BE (1972) A new translucent cement for dentistry. The glass ionomer cement. Br Dent J 132: 133-135.

7. Naasan MA, Watson TF (1998) Conventional glass ionomers as posterior restorations. A status report for the American Journal of Dentistry. Am J Dent 11: 36-45.

8. Berg JH (2002) Glass ionomer cements. Pediatr Dent 24: 430-438.

9. Sidhu SK, Nicholson JW (2016) A review of glass-ionomer cements for clinical dentistry. J Funct Biomater 7: 16.

10. Överbo RC, Raadal M (1999) Microleakage in fissure sealed with resin or glass ionomer cement. Scand J Dent Res 98: 66-69.

11. Lee HS, Berg JH, García-Godoy F, Jang KT (2008) Longterm evaluation of the remineralization of interproximal caries-like lesions adjacent to glass-ionomer restorations: A micro-CT study. Am J Dent 21: 129-132. 
12. Trairatvorakul C, Itsaraviriyakul S, Wiboonchan W (2011) Effect of glass-ionomer cement on the progression of proximal caries. J Dent Res 90: 99-103.

13. Kovarik RE, Muncy MV (1995) Fracture toughness of resin-modified glass ionomers. Am J Dent 8: 145-148.

14. Mount GJ (1999) Glass ionomers: A review of their current status. Oper Dent 24: 115-124.

15. Irie M, Nakai H (1988) Mechanical properties of silver-added glass ionomers and their bond strength to human tooth. Dent Mater J 7: 87-93.

16. Elsaka SE, Hamouda IM, Swain MV (2011) Titanium dioxide nanoparticles addition to a conventional glass-ionomer restorative: Influence on physical and antibacterial properties. J Dent 39: 589-598.

17. Garoushi S, Vallittu P, Lassila L (2017) Hollow glass fibers in reinforcing glass ionomer cements. Dent Mater 33: e86-e93.

18. Miki S, Kitagawa H, Kitagawa R, Kiba W, Hayashi M, et al. (2016) Antibacterial activity of resin composites containing surface pre-reacted glass-ionomer (S-PRG) filler. Dent Mater 32: 1095-1102.

19. Yoneda M, Suzuki N, Masuo Y, Fujimoto A, lha K, et al. (2012) Effect of S-PRG elute on biofilm formation and enzyme activity of oral bacteria. Int J Dent 2012: 814913.

20. Suzuki N, Yoneda M, Haruna K, Masuo Y, Nishihara T, et al. (2014) Effects of S-PRG eluate on oral biofilm and oral malodor. Arch Oral Biol 59: 407-413.

21. Hahnel S, Wastl DS, Schneider-Feyrer S, Giessibl FJ, Brambilla E, et al. (2014) Streptococcus mutans biofilm formation and release of fluoride from experimental resin-based composites depending on surface treatment and S-PRG filler particle fraction. J Adhes Dent 16: 313-321.

22. Fujimoto $Y$, Iwasa M, Murayama R, Miyazaki M, Nagafuji A, et al. (2010) Detection of ions released from S-PRG fillers and their modulation effect. Dent Mater J 29: 392-739.

23. An J, Park H, Seo H, Lee S (2015) Antibacterial properties of pit and fissure sealant containing S-PRG filler on Streptococcus mutans. J Korean Acad Pediatr Dent 42: 302-311.

24. Lucas ME, Arita K, Nishino M (2003) Toughness, bonding and fluoride-release properties of hydroxyapatite-added glass ionomer cement. Biomaterials 24: 3787-3794.

25. Arita K, Yamamoto A, Shinonaga Y, Harada K, Abe Y, et al. (2011) Hydroxyapatite particle characteristics influence the enhancement of the mechanical and chemical properties of conventional restorative glass ionomer cement. Dent Mater J 30: 672-683.

26. Shinonaga Y, Arita K, Nishimura T, Chiu SY, Chiu HH, et al (2015) Effects of porous-hydroxyapatite incorporated into glass-ionomer sealants. Dent Mater J 34: 196-202.

27. Shinonaga Y, Arita K (2012) Antibacterial effect of acrylic dental devices after surface modification by fluorine and silver dual-ion implantation. Acta Biomater 8: 1388-1393.

28. Hatibovic-Kofman S, Koch G, Ekstrand J (1997) Glass ionomer materials as a rechargeable fluoride-release system. Int J Paediatr Dent 7: 65-73.

29. Lewis SM, Coleman NJ, Booth SE, Nicholson JW (2013) Interaction of fluoride complexes derived from glass-ionomer cements with hydroxyapatite. Ceram Silik 57: 196-200.

30. Chiu HH, Shinonaga Y, Nishimura T, Harada K, Arita K (2016) Behavior of trace elements in novel apatite-ionomer cement. Nano Biomed 8: 101-111.
31. Chiu SY, Shinonaga Y, Abe Y, Harada K, Arita K (2017) Influence of porous spherical-shaped hydroxyapatite on mechanical strength and bioactive function of conventional glass ionomer cement. Materials (Basel) 10: 27.

32. McCann HG (1969) The effect of fluoride complex formation on fluoride uptake and retention in human enamel. Arch Oral Biol 14: 521-531.

33. Putt MS, Kleber CJ (1985) Dissolution studies of human enamel treated with aluminum solutions. J Dent Res 64: 437-440.

34. Kleber CJ, Putt MS (1985) Uptake and retention of aluminum by dental enamel. J Dent Res 64: 1374-1376.

35. Höök M, Christoffersen J, Christoffersen MR, Leonardsen ES, Rassing MR, et al. (1994) Effects of aluminum (III) and fluoride on the demineralization of bovine enamel: A longitudinal microradiographic study. Scand J Dent Res 102: 198-201.

36. Yokel RA, McNamara PJ (2001) Aluminum toxicokinetics: An updated minireview. Pharmacol Toxicol 88: 159-167.

37. Nicholson JW, Czarnecka B (2009) Review paper: Role of aluminum in glass-ionomer dental cements and its biological effects. J Biomater Appl 24: 293-308.

38. Besinis A, van Noort R, Martin N (2012) Infiltration of demineralized dentin with silica and hydroxyapatite nanoparticles. Dent Mater 28: 1012-1023.

39. Brudevold F, Tehrani A, Attarzadeh F, Goulet D, van Houte $\mathrm{J}$ (1985) Effect of some salts of calcium, sodium, potassium, and strontium on intra-oral enamel demineralization. $J$ Dent Res 64: 24-27.

40. Featherstone JD, Shields CP, Khademazad B, Oldershaw MD (1983) Acid reactivity of carbonated apatites with strontium and fluoride substitutions. J Dent Res 62: 1049-1053.

41. Shiozawa M, Takahashi $H$, Iwasaki $N$, Wada T, Uo $M$ (2014) Effect of immersion time of restorative glass ionomer cements and immersion duration in calcium chloride solution on surface hardness. Dent Mater 30: e377-e383.

42. Hotta M, Morikawa T, Tamura D, Kusakabe S (2014) Adherence of streptococcus sanguinis and streptococcus mutans to saliva-coated S-PRG resin blocks. Dent Mater J 33: 261-267.

43. Kaga M, Kakuda S, Ida $Y$, Toshima H, Hashimoto M, et al. (2014) Inhibition of enamel demineralization by buffering effect of S-PRG filler-containing dental sealant. Eur J Oral Sci 122: 78-83.

44. Mukai $Y$, Kamijo K, Fujino F, Hirata $Y$, Teranaka $T$, et al. (2012) Effect of denture base-resin with prereacted glass-ionomer filler on dentin demineralization. Eur $\mathrm{J}$ Oral Sci 117: 750-754.

45. Saku S, Kotake H, Scougall-Vilchis RJ, Ohashi S, Hotta M, et al. (2010) Antibacterial activity of composite resin with glass-ionomer filler particles. Dent Mater J 29: 193-198.

46. Yoshihara K, Nagaoka N, Maruo Y, Sano H, Yoshida Y, et al. (2017) Bacterial adhesion not inhibited by ion-releasing bioactive glass filler. Dent Mater 33: 723-734. 